\title{
Strengthening Science Vocabulary through the Use of Imagery Interventions with College Students
}

\author{
Marisa T. Cohen \\ Psychology Department, St. Francis College, Brooklyn, USA \\ Email: mcohen@sfc.edu
}

Received October $5^{\text {th }}$, 2012; revised November $6^{\text {th }}$, 2012; accepted November $16^{\text {th }}, 2012$

\begin{abstract}
This study was an extension of previous work designed to examine the effect of imagery on science vocabulary learning. One hundred students enrolled in a private college in Brooklyn, New York were randomly assigned to four different interventions: Word Only, Picture Presentation, Image Creation-No Picture, and Image Creation-Picture. These interventions were developed taking into account the ability of images to facilitate vocabulary learning, the dual coding theory, and depth of processing. Results demonstrated that students in the imagery creation groups (Image Creation-No Picture and Image Creation-Picture) scored higher on the outcome measures than students placed in the Word Only intervention at immediate recall. However, there were no significant differences shown among the imagery treatments or at delayed recall. The outcome scores from each group also followed the pattern predicted in that the deeper the students processed the "to be learned" vocabulary words, the more words they were able to acquire and retain. This work extends the previous research and highlights the benefits of vocabulary instruction using imagery at all instructional levels.
\end{abstract}

Keywords: Vocabulary Instruction; Science Literacy; Imagery; Dual Coding; Depth of Processing

\section{Literature Review}

Given that the benefits of integrating science instruction and literacy have been well documented, and the push for developing a scientifically competent society is ever so present (McKee \& Ogle, 2005; National Research Council, 1996; Thier \& Daviss, 2002; Yore, Hand, \& Prain, 2002), there is a great need for literacy-based interventions within the science content area. In order for students to learn complex science material, they must understand the terminology required so that they can speak and communicate using the language of science.

Learning new content area material is very difficult without a mastery of certain literacy skills, such as reading comprehendsion and vocabulary. A student cannot be expected to understand novel concepts without a firm grasp of the basic terminology used. Therefore, students must be instructed using effective interventions focused on building and strengthening their vocabularies. This will allow them to further their quest for knowledge in the discipline, anchored by a strong understanding of the integral components needed.

\section{Science and Literacy}

McKee and Ogle (2005) broadly define literacy as “...the ability to use reading and writing, speaking and listening sufficiently well to engage in thinking and to communicate ideas clearly. [It also incorporates]...the ability to critically analyze and evaluate information...” (p. 2). McKee and Ogle (2005) stress the importance of vocabulary knowledge, a key component of literacy, which is the ability to think about and understand the meanings of words. In order to deeply think about material, one must understand the content specific terminology that identifies the ideas or phenomena presented.

McKee and Ogle (2005) note that literacy and science work together to strengthen and clarify learning in each respective domain, motivate students to understand what is going on around them, and help students develop a desire to learn the meanings of new words and understand their uses. The stronger a student's literacy skills, the better they grasp science material.

Thier and Daviss (2002) state that scientific understanding is accessed through language, and effective science teaching and learning depend on joining strong language skills with science to communicate meaning in the context of the physical world. The marriage of literacy and science also enables individuals to confront questions that require the use of scientific thinking and understanding of scientific information. Language is necessary for both creating and conveying knowledge. Yore et al., (2003) note that language is a means for "doing" science and constructing understandings. It is also an end whereby procedures and scientific understandings are relayed to others.

\section{Vocabulary and Its Importance for Science Instruction}

While a good command of vocabulary is necessary for all subjects, its effects on learning are all the more apparent in content areas such as science. Science has its own language, in which students are introduced to either completely new words or novel uses of familiar words. Wilson (1998) states that a particular scientific word should be taught to students at the appropriate time, such as when it will fill a gap in the children's knowledge and when they have a clear need to use appropriate vocabulary to describe something that has been experienced. Wilson claims that if executed correctly, word learning can enhance science learning. This in turn will facilitate the command of more complex language skills such as speaking, reading, and writing. 
When examining vocabulary interventions and techniques to facilitate comprehension, it is important to discuss the formation of images, or mental representations, by students. The creation of images while one reads text serves as an aid to understanding and remembering (Sadoski, Goetz, \& Kangiser, 1988). Individuals asked to create mental images of events described in sentences learn two to three times as much as those who just read the sentences aloud (Anderson, 1971). Imagery is a major component of cognitive processing, and as such warrants it own detailed discussion.

Students told to create images have been shown to learn new material more efficiently. In a study by Anderson and Kulhavy (1972) using high school seniors, comprehension increased with the amount of imagery the students reported. In this study, 63 seniors were instructed to form mental images while reading a textbook passage. Students were assessed as to their comprehension abilities by a combined short answer and multiple choice test. A follow-up questionnaire indicated that more than half of the control group employed imagery on their own while studying the passage, which confounded the results. Also, one third of the group instructed to form images did not do so. Possibly due to the lack of fidelity to treatment, results failed to show a difference between the group instructed to form images and the one just instructed to read the text. However, the students from both groups who formed images outperformed those who did not.

One theory which examines the use of imagery in the processing of information, and has been empirically tested by numerous studies is dual coding. Paivio's dual coding theory states that information is processed along two distinct channels: verbal and visual. Research has shown that memory for verbal information is enhanced when the information is accompanied by a visual presentation, either real or imagined (Anderson \& Bower, 1973). The dual coding theory links vocabulary to comprehension through the use of images in improving understanding and enabling deeper processing. As such, this theory was used to frame the design of the vocabulary interventions in the present study.

\section{Present Study}

In order to test the assumptions of the dual coding theory, as well as extend the author's previous research, the effects of various manipulations with regard to image presentation and image creation on word learning and retention were examined using a group of college students. Four different interventions altering how vocabulary was presented were used: a Word Only method, which involved the simple verbal presentation of the word; a Picture Presentation, in which the vocabulary was paired with a picture; an Image Creation-No Picture intervention, in which students were told to draw a visualized image on paper; and an Image Creation- Picture intervention which was similar to the previous image creation intervention, but added a visual prompt of a picture.

\section{Hypotheses}

Based on the theory of dual coding, it was hypothesized that students instructed via any of the imagery interventions, would demonstrate better mastery of the vocabulary than when presented with only the word, because adding an imagery component facilitates vocabulary learning (Sadoski, 2005). There- fore, it was predicted students would demonstrate better vocabulary acquisition after the Picture Presentation, Image Creation-No Picture, and Image Creation-Picture interventions compared to the Word Only intervention. Furthermore, it was hypothesized that the imagery interventions would aid the students in remembering the vocabulary words presented to them, acquiring their meaning, as well as retaining this knowledge over time.

Creating images, especially by drawing them, increases the depth at which students process information and the meanings of words. This is because "... drawing requires careful observation of an object's or phenomenon's distinctive characteristics [which enables] students to attend to details they might otherwise overlook" (Armon \& Morris, 2008: p. 49). Therefore, according to depth of processing, the Image Creation-No Picture intervention should be the most effective, as it required the students to process the "to be learned" information at the deepest level. This is because the participant must become active in creating an image and drawing it on paper. The Image Creation-Picture intervention should be the second most effective treatment because it also required deep processing, however the picture was provided. The Picture Presentation intervention should be the third most effective, as imagery was employed; however, the level of processing was not as deep as the aforementioned interventions because the pictures were presented to the students. The least effective strategy for vocabulary learning should be the Word Only presentation, as the word was only presented in one code: verbal, only allowing for shallow processing.

Finally, it was hypothesized that student responses to assessments created with the aim of determining how useful the interventions were would mimic the quantitative results. These responses should match the pattern predicted in the depth of processing hypothesis, with those in the image creation interventions reporting the greatest benefits.

To summarize, there were four specific hypotheses set at the beginning of the study. The first hypothesis was that imagery would facilitate vocabulary acquisition. The second hypothesis was that the depth with which one processes the vocabulary influences vocabulary acquisition. The third hypothesis was that the interventions would enable students to retain the vocabulary over time and facilitate delayed recall of the words. The fourth and final hypothesis was that the qualitative results would mimic the quantitative data.

\section{Methodology}

\section{Participants}

Participants were recruited from five different psychology classes (General Psychology, Developmental Psychology, Statistics, and two research recitation classes) at a private college in Brooklyn Heights, New York. Data were collected from 100 students, 25 males and 75 females. The mean age of the students was 20.82 years.

In terms of ethnicity, $44 \%$ of the sample was Caucasian, $23 \%$ was Latino, $15 \%$ was African American, and the other $18 \%$ identified themselves as "Other" or a mix of the aforementioned categories. English was the first language learned by $76 \%$ of the students, and $54 \%$ of the sample spoke English at home. 


\section{Pre-Intervention Measures}

Development of target vocabulary. The earth science words were selected from the Earth Science Vocabulary List (Personalized Programming Service, n.d.), which contains 1610 earth science words needed for comprehension of this content area. The words chosen were all easily visualized nouns.

A definition for each word was generated from MerriamWebster's Collegiate Dictionary (1996), by simplifying these definitions to provide a clear understanding of the words. Sentences illustrating the use of the words accompanied these definitions, and were reviewed by a teacher independent of the study to be certain that they were appropriate for the subjects.

Selection of pictures. The pictures used for the Picture Presentation and Image Creation-Picture interventions were found through a Google image search. The pictures were printed in color, pasted onto 4 " $\times 6$ " index cards, and were laminated.

Demographic information. All consenting students were given a demographic information sheet. This enabled the researcher to collect information on characteristics of the participants that may have been useful in analyzing the results, such as age, gender, number of languages spoken, first language learned, and what language was spoken at home. Though no specific differences between the students, based on their demographics were hypothesized, the information collected enabled the researcher to examine if there were any unanticipated differences between the students.

Vocabulary pretest. A researcher designed vocabulary pretest was given to the students, containing 25 earth science words. The test consisted of the vocabulary words followed by four choices: three definitions and a "Not sure/Do not remember" choice. Students were instructed to select the correct definition of the word. Sixteen words which $80 \%$ or more of the students did not know were selected for the interventions.

Prior vocabulary knowledge. The SAT verbal score was obtained for each student in order to get a sense of their vocabulary abilities. Twenty percent of the students had no score on record, because they were transfer students or never submitted it with their application. These students were asked to provide the score in a self-report fashion.

\section{Interventions}

This study examined predictions based both on the dual coding theory. The various manipulations of imagery employed in the interventions created a between-subjects variable with four different levels.

Training condition. Before each intervention was carried out by the researcher, a training condition was conducted using the word "dog". This word was chosen as it is simple and familiar to the students, allowing them to focus on the type of task they would be engaging in, rather than on the definition of the word itself. Each group was instructed via the intervention they were assigned to. For example, the students who were assigned to Picture Presentation were shown a picture of a dog; the Image Creation-No Picture treatment participants were told to come up with an image of a dog in their minds and to draw it; the Image Creation-Picture treatment participants saw a picture of a dog and then drew it on paper; and the Word Only treatment participants heard the word dog repeated a second time.

Word Only intervention. For the Word Only treatment, a variation of the procedure used in a 1978 study by Hargis and Gickling (as presented in Sadoski, 2005) was used. The researcher first presented the participants with the word written on a 4 " $\times 6$ " index card, pronounced the word, provided the definition of the word, and gave the participants a sentence containing the word. Finally, the researcher presented the word on the index card again and repeated the word.

Picture Presentation intervention. For the Picture Presentation intervention, the aforementioned procedure was carried out. However, instead of repeating the word, in step six, the researcher presented a picture of the word. For example, if the word "school" was taught, a picture of a schoolhouse was provided on an index card.

Image Creation-No Picture intervention. The procedure used for the Picture Presentation intervention was repeated; however, instead of showing a picture, the researcher instructed the student to create a mental image of the word and to draw it on a sheet of $8 \frac{1}{2}$ " $\times 11$ " paper. The decision to have the participant draw the word was formulated to make the process active and to check to see that indeed an image was created in response to the word.

Image Creation-Picture intervention. The procedure used for the Image Creation-No Picture presentation was repeated; however instead of showing the participants the word written on an index card, they were presented with a picture of the word. This step was changed to help clarify what the definition meant, assuring that students would not form inaccurate images in their heads. Students were told that they did not need to copy the picture shown, but could use it to help guide them.

All four groups were shown the word, heard the word, given a definition, and had it presented in a sentence in the same sequence in order to equalize the treatments. The fifth and sixth steps varied between the interventions. See Table 1 for the format in which each intervention was carried out, which was used in the aforementioned Cohen and Johnson (in press) study. Also, in order to equalize the exposure to the words, the amount of time for the variable steps was held constant at 30 seconds. This means that the word and picture cards were held up for a total of 30 seconds and students were only given this amount of time in which to create their pictures before moving to the next word.

Table 1.

Format of the Interventions.

\begin{tabular}{|c|c|c|c|}
\hline Word Only & $\begin{array}{c}\text { Picture } \\
\text { Presentation }\end{array}$ & $\begin{array}{c}\text { Image } \\
\text { Creation- } \\
\text { No Picture }\end{array}$ & $\begin{array}{c}\text { Image } \\
\text { Creation- } \\
\text { Picture }\end{array}$ \\
\hline $\begin{array}{l}\text { 1.Word } \\
\text { presented on } \\
\text { index card } \\
2 \text {. Word } \\
\text { pronounced }\end{array}$ & $\begin{array}{l}\text { 1. Word } \\
\text { presented on } \\
\text { index card } \\
\text { 2. Word } \\
\text { pronounced }\end{array}$ & $\begin{array}{l}\text { 1. Word } \\
\text { Presented on } \\
\text { index card } \\
2 . \text { Word } \\
\text { pronounced }\end{array}$ & $\begin{array}{l}\text { 1. Word } \\
\text { presented on } \\
\text { index card } \\
2 . \text { Word } \\
\text { pronounced }\end{array}$ \\
\hline $\begin{array}{l}\text { 3. Definition } \\
\text { given } \\
4 \text {. Word used } \\
\text { in a sentence } \\
5 . \text { Word } \\
\text { presented } \\
\text { again } \\
6 \text {. Word } \\
\text { repeated }\end{array}$ & $\begin{array}{l}\text { 3. Definition } \\
\text { given } \\
4 \text {. Word used } \\
\text { in a sentence } \\
5 . \text { Word } \\
\text { presented } \\
\text { again } \\
6 \text {. Picture } \\
\text { presented }\end{array}$ & $\begin{array}{l}\text { 3. Definition } \\
\text { given } \\
4 \text {. Word used } \\
\text { in a sentence } \\
5 \text {. Word } \\
\text { presented } \\
\quad \text { again } \\
6 \text {. Student } \\
\text { created a } \\
\text { mental image } \\
\text { and drew it }\end{array}$ & $\begin{array}{l}\text { 3. Definition } \\
\text { given } \\
4 \text {. Word used in } \\
\text { a sentence } \\
5 \text {. Student } \\
\text { shown a picture } \\
\text { of the word } \\
6 \text {. Student } \\
\text { created a mental } \\
\text { image and drew } \\
\text { it }\end{array}$ \\
\hline
\end{tabular}




\section{Post-Intervention Measures}

Students' acquisition and retention of the science vocabulary was assessed by a battery of experimenter designed vocabulary comprehension tests. The measures were given a day after the instruction of the vocabulary words, to assess immediate recall, and two weeks later. The tests given two weeks after the vocabulary instruction enabled the researcher to determine if the interventions allowed for efficient retention of the vocabulary words. At each time point, the students' vocabulary knowledge was assessed using two measures: a word fill-in task and definition word match task.

Word fill-in task. The word fill-in tasks were similar to Cloze tests in that they consisted of simple sentences with blanks in place of the vocabulary words instructed. Students were given a series of sentences with one or two blanks for the earth science words. They were instructed to pick the word from the choices provided next to the blank in order to correctly fill in the sentence. This required students to not only remember the definitions of the vocabulary words they were taught, but also to select the specific words that made sense in the context of the sentences. The number of correct words filled in the blanks was added to determine the number of points awarded.

The delayed word fill-in tasks were comprised of the same word choices as the immediate recall fill-in tasks, but the content of the sentences was slightly altered to prevent students from remembering their answer choices.

Definition word match. All 16 words instructed were incorporated in the definition word match tasks. Students received two word matches, each with eight words for the earth science terms. On the left side of the page, students were presented with all eight vocabulary words. On the right side of the page, they were provided with the definitions of the words, in a rearranged format. Students were instructed to select the definition that corresponded to each word.

The delayed definition word match tasks were rearranged, by mixing up the groups of words, to prevent any repeated testing effects.

\section{Vocabulary Learning Evaluations}

Upon completion of the delayed recall measures, each student was given an evaluation form. This was used to elicit students' opinions about the intervention he/she was exposed to.

\section{Research Design}

A quasi-experimental design was used for the purpose of this study. While students were randomly assigned to each intervention, they could not be randomly assigned to classes, as these were intact groups.

The independent variable was the interventions, which consisted of four "levels". These levels varied in terms of the manipulations with regards to image presentation and image creation. The way in which the manipulations were manifested within the interventions, as in the researchers previous study, is shown by Figure 1.

The dependent variable in this study was vocabulary comprehension, which was measured by two different tasks: word fill-in and definition word match at two different time points: both immediately after the interventions and two weeks later, to examine delayed recall. Therefore, this study also employed a 2 (outcome task: word fill-in, definition word match) $\times 2$ (time point: immediate recall, delayed recall) within-subjects design.

\section{Procedure}

The researcher visited all classes and randomly assigned the participating students numbers ranging from 1 to 100 . After this, the participants took the earth science vocabulary pretest. They were instructed to record their numbers on the upper left hand corners of the papers, and were allowed approximately $30 \mathrm{~min}-$ utes to complete these tests. After one class was finished, the researcher rotated through the remaining classes, so all of the pretests could be analyzed together.

Once all of the pretests were scored, the researcher selected 16 earth science vocabulary words with which $80 \%$ or more of the students were unfamiliar. These words were used for the interventions and outcome measures.

Within each class, students were randomly assigned to one of the four interventions by signing up for vocabulary training appointments with the researcher. Four time slots were offered to each class, and each slot was randomly assigned to each of the four interventions. Students were to meet the researcher in a laboratory during the time slot they signed up for and were instructed on the earth science terms as per the intervention they were randomly assigned to.

Twenty-four hours after the instruction of the terms, the researcher returned to the class and administered the earth science immediate recall outcome measures. She gave the students the earth science fill-ins and word matches, and instructed them to record their identification numbers on the papers. Participants were given 10 to 15 minutes to complete these assessments.

Two weeks after the initial vocabulary instruction, the researcher returned with the delayed outcome measures to assess retention of the earth science vocabulary words. The same procedure used for the immediate recall outcome measures was employed.

After the words were instructed and assessed using the comprehension measures, the researcher visited the class to give the students the vocabulary learning evaluations. Students were given 10 minutes to complete this assessment.

\section{Data Analyses}

Statistical analyses enabled the researcher to determine how effective the interventions were in improving the participants' science vocabulary acquisition and retention. Descriptive statistics were computed for each outcome measure and the verbal SAT scores to examine the means and standard deviations, as well as identify any outliers.

Examination of the relationships between the variables. Demographic variables, such as age, gender, ethnicity, number

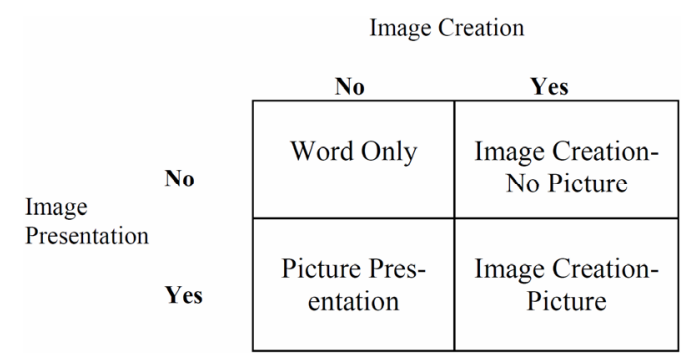

Figure 1.

Layout of the interventions. 
of older or younger siblings, and number of languages spoken, were entered into a general linear model to determine if any were significant predictors of the outcome scores.

Benefits of imagery and deep levels of processing (Hypotheses 1, 2, and 3). To test the first two hypotheses, as well as the third regarding the benefits of imagery as well as deep levels of processing, a series of statistical tests was carried out. A one-way analysis of covariance (ANCOVA) was used to examine any differences between the students in the intervenetion groups for fill-in and word match tasks, using the composite score. This test was conducted at both the immediate and delayed recall time points. The verbal SAT scores were used as a covariate in the statistical model. Pairwise comparisons were also conducted to determine where any observed statistical differences lie.

Participants' perceptions regarding the utility of the interventions (Hypothesis 4). To examine the fourth hypothesis focusing on the utility of the interventions, the first four questions of the vocabulary learning evaluation form were coded to represent the answers the students provided. Numbers were assigned to the answer choices in the following way: $3=$ completely agree, 2 = somewhat agree, 1 = somewhat disagree, and 0 = completely disagree. An analysis of covariance (ANCOVA) was carried out for each question to explore any differences between students' perceived usefulness of the interventions and the particular intervention they were assigned to.

\section{Results}

\section{Demographics}

Data were collected from 100 students. Thirteen of these students were in the researcher's General Psychology class, 28 in Developmental Psychology, 37 in the two Research Methods recitations combined, 20 in Statistics, and an additional 2 students who were both in the Developmental and one of the Research Methods recitation classes. There were 75 females in this sample and 25 males.

The mean age of the students was 20.82 years, with a range from 18 to 42 years of age. The mean number of older siblings was $0.91(S D=1.22)$, ranging from 0 to 5 ; for younger siblings it was $1.03(S D=1.43)$, ranging from 0 to 8 . The mean verbal SAT score, from the 88 students for whom it could be provided for was $480.34(S D=78.12)$, with a minimum of 290 and a maximum of 660 .

The breakdown of ethnicity is as follows: $44 \%$ Caucasian/ White, 15\% African American, 23\% Hispanic, 18\% identified themselves only as "Other”. In terms of first language learned, $76 \%$ spoke English first, and for 9\% it was Spanish. For language spoken at home, 54\% spoke English, and 19\% spoke English and Spanish in the home. The remaining 27\% was distributed amongst a variety of other languages.

\section{Influence of Demographic Variables}

Looking at all the aforementioned demographic variables at immediate recall, by entering them into a general linear model to screen for any potential demographic predictors, correcting for the covariate and intervention assigned, only these two variables reached significance at immediate recall; verbal SAT: $F(8.65)=5.85, p=0.02$; intervention: $F(8.65)=5.02, p=0.03$. At delayed recall, it was shown that the number of younger siblings had a significant effect $F(1.74)=4.73, p=0.03$. This effect remained even when the intervention and verbal SAT score were added in to the equation $F(1,64)=4.14, p=0.05$. Examining this relationship more closely, it was shown that as the number of younger siblings increased, the composite score on the delayed outcome measures also increased $r(81)=0.22, p$ $=0.05$.

Significant correlations were shown between verbal SAT score and immediate recall composite outcome measures $r(74)=$ $0.36, p=0.00$, and between verbal SAT score and the delayed recall composite outcome measures $r(73)=0.65, p=0.00$.

\section{Acquisition Results}

An analysis of covariance (ANCOVA) was conducted to compare the effectiveness of the four interventions in improving the vocabulary acquisition of the students at immediate recall. The independent variable was the intervention, and the dependent variable was the composite outcome score of the two outcome measures at immediate recall. Participants' verbal SAT scores were used as the covariate. There was a significant difference shown between the interventions $F(3.69)=3.38, p=$ 0.02 . The means followed the pattern predicted by the hypotheses: Image Creation-No Picture $(M=27.40, S D=3.65)$, Image Creation-Picture $(M=25.41, S D=4.26)$, Picture Presentation $(M=22.47, S D=8.05)$, and Word Only $(M=20.17$, $S D=6.20)$.

With respect to the interventions, follow-up comparisons among the four intervention conditions indicated that college students assigned to the Word Only intervention demonstrated a statistically significant difference from those in the Image Creation- No Picture $(p=0.00)$ and Image Creation-Picture presentation $(p=0.00)$. The students in the group which did not employ imagery scored the lowest. See Table 2 for the results of the comparisons.

This data support the first hypothesis in that students assigned to the image creation interventions (Image Creation- No Picture and Image Creation-Picture) significantly outperformed those in the Word Only intervention. There is only partial support for the second hypothesis with regards to depth of processing, being that the pattern was as predicted, however results did not demonstrate significant differences between each and every intervention.

\section{Retention Results}

A second ANCOVA was carried out to examine retention, this time using the delayed recall composite score as the dependent variable. Participants' verbal SAT scores were once again used as the covariate. There was no significant effect of the intervention on the retention of the vocabulary words, however the means followed the pattern predicted by the hypothesis: Image Creation-No Picture $(M=26.75, S D=6.12)$, Image Creation-Picture $(M=25.24, S D=5.506)$, Picture Presentation $(M=23.05, S D=5.71)$, and Word Only $(M=21.71, S D=$ 7.91). Overall, there was no support for the third hypothesis.

\section{Results from the Vocabulary Learning Evaluations}

With regards to the vocabulary learning evaluation forms, there were no statistically significant differences between the groups in terms of their self-rated abilities to learn the words ( $p$ $=0.24)$, remember the words $(p=0.83)$, how useful they thought the interventions were $(p=0.49)$, how fun they thought 
Table 2.

Immediate recall comparisons.

\begin{tabular}{|c|c|c|c|c|c|c|}
\hline \multicolumn{7}{|l|}{ Multiple Comparisons } \\
\hline \multicolumn{7}{|c|}{ Immediate Recall Composite Score } \\
\hline \multicolumn{7}{|l|}{ Tukey HSD } \\
\hline \multirow{2}{*}{ (I) Intervention Assigned } & \multirow{2}{*}{ (J) Intervention Assigned } & \multirow{2}{*}{ Mean Difference (I-J) } & \multirow{2}{*}{ Std. Error } & \multirow{2}{*}{ Sig. - } & \multicolumn{2}{|c|}{ 95\% Confidence Interval } \\
\hline & & & & & Lower Bound & Upper Bound \\
\hline \multirow{3}{*}{ Word Only } & Picture Presentation & -3.50 & 1.78 & 0.21 & -8.16 & 1.16 \\
\hline & Image Creation- No Picture & $-7.85^{*}$ & 1.84 & 0 & -12.68 & -3.03 \\
\hline & Image Creation- Picture & $-6.09^{*}$ & 1.87 & 0.01 & -10.98 & -1.19 \\
\hline \multirow{3}{*}{ Picture Presentation } & Word Only & 3.50 & 1.78 & 0.21 & -1.16 & 8.16 \\
\hline & Image Creation- No Picture & -4.36 & 1.82 & 0.09 & -9.13 & 0.42 \\
\hline & Image Creation- Picture & -2.59 & 1.85 & 0.50 & -7.43 & 2.26 \\
\hline \multirow{3}{*}{ Image Creation- No Picture } & Word Only & $7.85^{*}$ & 1.84 & 0 & 3.03 & 12.68 \\
\hline & Picture Presentation & 4.36 & 1.82 & 0.09 & -0.42 & 9.13 \\
\hline & Image Creation- Picture & 1.77 & 1.908 & 0.791 & -3.24 & 6.77 \\
\hline \multirow{3}{*}{ Image Creation- Picture } & Word Only & $6.09^{*}$ & 1.87 & 0.01 & 1.19 & 10.98 \\
\hline & Picture Presentation & 2.59 & 1.85 & 0.50 & -2.26 & 7.43 \\
\hline & Image Creation- No Picture & -1.77 & 1.91 & 0.79 & -6.77 & 3.24 \\
\hline
\end{tabular}

Based on observed means.

The error term is Mean Square (Error) $=35.470$.

*The mean difference is significant at the 0.05 level.

the interventions were $(p=0.08)$, or in the number of students who needed to use another method besides the intervention that they were assigned to in order to remember the words ( $p=$ 0.41 ). Based on the aforementioned data, there is no support for the fourth hypothesis in that the qualitative results do not mimic the quantitative results.

\section{Discussion}

\section{Influence of Demographic Variables}

It is important to note that the number of younger siblings influenced the outcome scores at delayed recall; more specifically, as the number of younger siblings increased within a family, the composite score on the delayed outcome measures increased. While this relationship was not examined by this particular study, this effect could be due to the practice individuals have with vocabulary memory methods as a result of aiding younger siblings in this task. However, without further investigation this is merely speculation.

\section{Imagery}

Hypotheses 1 and 3 focused on the use of imagery in facilitating the acquisition and retention of science content vocabulary by the learners. It was predicted that those in the imagery intervention groups would score significantly higher on the outcome measures at both time points, compared to the students in the Word Only intervention.

Based on the pairwise comparisons, the participants in the
Word Only group scored significantly lower than the students in the two image creation groups (Image Creation-No Picture and Image Creation-Picture) at immediate recall. Imagery, more specifically image creation, is an important component of vocabulary interventions. At delayed recall there were no significant differences shown, which suggests that these interventions may not be strong enough to aid a college age sample in remembering words over time. Perhaps with greater exposure to the vocabulary words, and more opportunities to illustrate their meaning, these interventions would have significantly impacted the students' learning over time.

Overall, after examining the results of the immediate and delayed recall outcome measures, hypothesis 1 was supported while hypothesis 3 was not. Imagery was shown to facilitate the acquisition of science vocabulary learning, but it did not aid in the retention of these words. However, it is important to note that while there were no effects as a result of imagery in support of hypothesis three, some trends were witnessed as a result of depth of processing.

\section{Depth of Processing}

Hypotheses 2 and 3 related to depth of processing and its potential effects in facilitating the acquisition and retention of the science content words. It was hypothesized that the Image Creation-No Picture intervention would help the students acquire and retain the novel vocabulary words the best. This is because this intervention affords the students the deepest level of processing, as they must create a picture which clearly illustrates the 
meaning of the word, without any assistance in the form of a visual prompt. While this intervention was anticipated to be the most effective in this particular study, in previous works (Cohen \& Johnson, in press), this was not the case. This is hypothesized to be a developmental issue. In an earlier version of this study, focusing on fifth grade biology learners, Image Creation-Picture was predicted to be the most efficient intervention in terms of word acquisition and retention. Image Creation-Picture, when compared to the Image Creation-No Picture intervention, helps to clarify the meanings of the vocabulary words, as students are presented with the images first. For Image Creation-No Picture, the student must focus on understanding and learning the definition, as well as creating a plan for drawing a picture of the word without any visual prompt. This may be easier for older students such as those included in this study, but such an active and cognitively difficult strategy may not be productive for the younger students. The trends shown by the Cohen and Johnson study (in press) suggest this aforementioned pattern with the younger students.

In this particular study, the trends were as predicted in terms of the composite outcome scores at both immediate and delayed recall. Students in the Image Creation-No Picture intervention scored the highest, followed by those in the Image CreationPicture group, the Picture Presentation group, and finally the Word Only group. While differences were not significant between each group, the order of this data suggest that possibly with more challenging words or a larger sample, these results may show significance.

\section{Students' Perceptions of the Interventions}

Vocabulary learning evaluations. While the vocabulary learning evaluations brought in a qualitative piece to the study, the questions were quantitatively analyzed. Analyses of covariance were used for the first five questions of these forms.

It was interesting that no differences were found between the intervention groups in terms of their perceptions of their ability to learn the words, remember the words, the utility of the interventions, and how fun they perceived them to be. In addition, no differences were found between the students exposed to the different interventions in terms of how many used another method to help them remember the words as asked by question 5 .

However, this is not all that surprising. The intervention evaluation form was given to the students two weeks after the initial instruction of the vocabulary words. Perhaps too much time passed in order for the students to accurately remember how they learned the words and the specifics of the interventions to which they were exposed.

During the interventions themselves, many students were eager to describe the benefits of the condition they were exposed to. Many participants discussed how helpful the incorporation of images was to them. One participant from the Picture Presentation group said, "What I remembered was purely from meeting once with you. Seeing the pictures helped because I'm more of a visual experience or hands on learner. The pictures helped a lot.”

Some students who used imagery also employed additional strategies to facilitate their acquisition and recall of the terms. One student in the Image Creation-Picture group noted, "For the word mesa, I remembered it because in Spanish that means table. It's a flat surface. Cumulus, I remembered because it accumulates water." Other students described using techniques such as repetition of the words during the initial instruction phase and process of elimination during the outcome measures.

Despite these positive statements regarding the interventions, hypothesis 4 was not supported by the results of the evaluation forms. The students' answers did not reflect those shown by the quantitative results. Perhaps if students were given more time and open-ended questions, they could have been more explicit as to their perceptions of the interventions and expressed their ideas about vocabulary learning more clearly.

\section{Overall Discussion}

The first hypothesis was supported in that imagery facilitated vocabulary acquisition. The second hypothesis was only partially supported in that the trends were as predicted with regards to depth of processing, however differences between the interventions failed to show significance. The third hypothesis was not supported, being that there were no differences found between the imagery and no imagery interventions at retention, however trends once again followed what was predicted as a result of depth of processing. The fourth and final hypothesis regarding the qualitative results also lacked evidence.

Overall, this study lent some support to the utility of images and deep levels of processing. This study provided a way to connect abstract psychological concepts with actual classroom practice. These theories are worth examining further so as to devise more effective interventions to implement in the classroom with this age group of students.

\section{Limitations}

While great care was taken to reduce as many confounding variables as possible, there were limitations to this study. While these limitations may not have affected the results in any major way, they are important to discuss.

The individual differences of the participants, which included their prior knowledge and abilities, may have influenced the results. In terms of prior knowledge, vocabulary words which centered on content familiar to certain participants may have been more easily remembered by them. The use of the verbal SAT scores as a covariate was able to counter this limitation to an extent by ascertaining the degree of the participants' prior vocabulary knowledge. Selecting words which $80 \%$ or more of the students were unfamiliar with, also removed some of influence of individual differences, as it was less likely that the students had experienced the words before. Individual differences exert confounding effects on many vocabulary and imaging studies.

As was evidenced by the Anderson and Kulhavy study (1972), it is difficult to assume that the participants were not forming images on their own, even if they were not explicitly told to do so. In the aforementioned study, analysis of a postexperimental questionnaire demonstrated that a majority of those in the control group consisting of high school seniors reported forming images during reading. Perhaps in the current study, students used strategies to memorize the vocabulary words besides those which were presented to them in their intervention condition. Even though students were told to use the interventions provided, this cannot be entirely prevented or controlled.

Finally, the results of this study may not apply to vocabulary words from all disciplines and content areas. Requiring students 
to create their own images may not be possible in all situations. As per the dual coding theory, visual imagery may not work as effectively for abstract words. The concreteness of the vocabulary on which one is instructed plays a major role in the ability of students to create imagery and to learn it. Further research must be done to determine when dual coding is valuable as an intervention, and when it is not.

\section{Future Research}

In future studies it would be beneficial to separate results in terms of the quality and quantity of memory to see how effective the interventions actually are. For example, quality is the thoroughness with which information is remembered, such as how detailed the definitions are upon recall. The quantity of memory focuses on how much is remembered, or how many definitions can be recalled, despite their total accuracy. While this study examined quantity, it did not look at quality. Perhaps measures in which students are instructed to write the definitions from memory should be incorporated into future research. These definitions could then be studied in terms of the level of understanding and specificity they convey.

Research should also aim to assess the image evoking ability of various types of words and texts. This would enable curriculum designers and textbook publishers to implement interventions in content areas in which little to no imagery is evoked by the reading materials.

\section{Educational Implications}

This study extended work by the author in ascertaining whether the interventions which had previously been proven to improve the vocabulary learning of fifth grade students would be appropriate and beneficial for a college aged sample. While results were modest, students seemed to enjoy the exercise and those in the image creation imagery interventions did show a marked improvement over those in the Word Only intervention at immediate recall.

Even though significant differences were not witnessed between every single intervention on the pairwise comparisons, this study has important implications. Students were instructed on only 16 vocabulary words and were tested both the day after instruction and two weeks later. As research has shown, perhaps multiple exposures to the vocabulary would have led to even more significance.
Regardless of which type of intervention is implemented, teachers must remember to stress the importance of vocabulary learning and comprehension strategies and incorporate them in content area learning. This will serve both to further assist those who are doing well in continuing to learn, and to bridge the achievement gap between students.

\section{REFERENCES}

Anderson, R. C. (1971). Encoding processes in the storage and retrieval of sentences. Journal of Experimental Psychology, 91, 338-341. doi: $10.1037 / \mathrm{h} 0031833$

Anderson, J. R., \& Bower, G. H. (1973). Human associative memory. Washington DC: Winston.

Anderson, R. C., \& Kulhavy, R. W. (1972). Imagery and prose learning. Journal of Educational Psychology, 63, 242-243. doi: $10.1037 / \mathrm{h} 0032638$

Cohen M. T., \& Johnson, H. L. (2012). Improving the acquisition and retention of science material by fifth grade students through the use of imagery interventions. Instructional Science, in press.

McKee, J., \& Ogle, D. (2005). Integrating instruction: Literacy and science. New York: Guilford Press.

Merriam-Webster's Collegiate Dictionary (1996). Springfield, MA: Merriam-Webster, Incorporated.

National Research Council, National Committee on Science Education Standards and Assessment (1996). National science education standards. Washington DC: National Academy Press.

Personalized Programming Service, Inc. (2011) Earth science vocabulary. URL (last checked 1 October 2009).

http://www.ppsisoft.com/pdf\%5cvocabearthscience.pdf

Sadoski, M. (2005). A dual coding view of vocabulary learning. Reading \& Writing Quarterly, 21, 221-238. doi:10.1080/10573560590949359

Sadoski, M., Goetz, E. T., \& Kangiser, S. (1988). Imagination in story response: Relationships between imagery, affect, and structural importance. Reading Research Quarterly, 23, 320-336. doi:10.2307/748045

Thier, M., \& Daviss, B. (2002). The new scienceliteracy: Using language skills to help students learn science. Portsmouth, NH: Heinemann.

Wilson, M. (1998). Identifying and teaching essential science vocabulary. School Science Review, 80, 63-66.

Yore, L. D., Bisanz, G. L., \& Hand, B. M. (2003). Examining the literacy component of science literacy: 25 years of language arts and science research. International Journal of Science Education, 25, 689-725. doi:10.1080/09500690305018

Yore, L. D., Hand, B. M., \& Prain, V. (2002). Scientists as writers. Science Education, 86, 672-692. doi:10.1002/sce.10042 